\title{
Consumers' Purchase Behavior Preference in E-Commerce Platform Based on Data Mining Algorithm
}

\author{
Wenjun Yang ${ }^{1}$, Jia Guo ${ }^{2 *}$ \\ ${ }^{1}$ School of E-commerce and Logistics Management, Henan University of Economics and Law, \\ Zhengzhou 450000, China \\ ${ }^{2}$ Department of Electronics and Information, Zhengzhou Sias University, Zhengzhou 450000, China \\ *Corresponding author: 20160134@huel.edu.cn
}

Received: July 14, 2021. Revised: December 24, 2021. Accepted: January 13, 2022. Published: January 14, 2022.

\begin{abstract}
E-commerce platform can recommend products to users by analyzing consumers' purchase behavior preference. In the clustering process, the existing methods of purchasing behavior preference analysis are easy to fall into the local optimal problem, which makes the results of preference analysis inaccurate. Therefore, this paper proposes a method of consumer purchasing behavior preference analysis on e-commerce platform based on data mining algorithm. Create e-commerce platform user portrait template with consumer data records, select attribute variables and set value range. This paper uses data mining algorithm to extract the purchase behavior characteristics of user portrait template, takes the characteristics as the clustering analysis object, designs the clustering algorithm of consumer purchase behavior, and grasps the common points of group behavior. On this basis, the model of consumer purchase behavior preference is established to predict and evaluate the behavior preference. The experimental results show that the accuracy rate of this method is $91.74 \%$, the recall rate is $88.67 \%$, and the $\mathrm{F} 1$ value is $90.17 \%$, which are higher than the existing methods, and can provide consumers with more satisfactory product information push.
\end{abstract}

Keywords - data mining algorithm, e-commerce platform, consumer purchase behavior, behavioral preference.

\section{INTRODUCTION}

Massive user behavior data contains great value. In addition to recommending a series of products that users may be interested in, famous e-commerce platforms such as Taobao and Jingdong also need to know which products users are most interested in and which products they are most likely to buy, because doing so will directly improve the conversion rate of users and the income of e-commerce platforms. Therefore, compared with the e-commerce platform, the user's purchase forecast has greater practical value [1]. In order to better push products to users, most e-commerce platforms require users to provide appropriate feedback information for the recommended content according to their own preferences or interests. According to the form of expression, they can be divided into explicit feedback and implicit feedback.

The main feature of explicit feedback data is that users express their preferences clearly, and this preference can be quantified. One of the most representative is the user's rating of the content, because the user knows that they are expressing their attitude, and gives a very clear attitude in a quantifiable way. Usually, the appropriate algorithm model is used to process the rating data, and finally the calculated results are pushed to each user. For e-commerce platforms, the most important channel to obtain scores is to collect users' comments on this purchase after purchasing goods. A typical example is the favorable comments and poor comments on Taobao. We have noticed that in order to collect explicit feedback, the e-commerce platform requires consumers to pay a certain time cost, which leads to the low efficiency of explicit feedback collection [2]. For most consumers, unless the shopping experience is affected to a certain extent, users generally will not choose bad reviews, but will choose default five-star reviews after confirming the receipt. And with the more and more sophisticated means of brushing orders and returning praise, the e-commerce platform is full of many false comments, which is extremely unfavorable for users to find the goods they are interested in and for the platform to push goods to consumers.

In this case, the advantage of implicit feedback is reflected. On the e-commerce platform, most of the feedback is the natural behavior left by users when they use the app, such as clicking, collecting, joining the shopping cart, purchasing and so on. In many cases, users will not score the purchased goods to show their preferences. The purpose of users leaving these data is not to tell their preferences, but we can "guess" their preferences from them, which is implicit feedback. Compared with explicit feedback, implicit feedback does not require users to have feedback operations other than 
browsing, and the process of obtaining user feedback is almost imperceptible, so it is easy to be accepted by users. For e-commerce platforms, the cost of obtaining implicit feedback is much lower [3]. Therefore, the research on implicit feedback has become an important topic of current e-commerce platform. Implicit feedback is actually the largest amount of data in the e-commerce platform. In order to mine valuable information from implicit feedback, it is necessary to conduct in-depth analysis of the data to obtain the user's purchase behavior preference, so as to predict the user's next purchase behavior. At present, some scholars have studied this. For example, Jiang $\mathrm{h}$ has studied a method for analyzing the purchase behavior of cigarette consumers based on C4.5 algorithm, and studied the purchase behavior of cigarette consumers by analyzing the algorithm of decision tree C4.5; Qian and $\mathrm{Xu}$ [4] studied a dynamic identification method of consumer brand decision preference based on machine learning, but the above methods did not fully consider the user portrait, resulting in low analysis accuracy and poor effect. Based on this, this paper introduces the data mining algorithm into this field and proposes an analysis method of consumer purchase behavior preference on the e-commerce platform. It can improve the satisfaction of consumers on the e-commerce platform and promote the development of the e-commerce platform.

\section{ANALYSIS OF CONSUMERS' PURCHASE BEHAVIOR}

\section{PREFERENCE IN E-COMMERCE PLATFORM BASED ON}

\section{DATA MINING ALGORITHM}

\section{A. Creating User Portrait Template of E-commerce Platform}

In order to analyze the consumer's preference of e-commerce platform, the user portrait template is created. In the era of big data, fragmented behavior tracks of consumers such as browsing, clicking, leaving messages and comments on the Internet are collected and stored. These fragmented data stored in the database can be used by the e-commerce platform to completely reconstruct the needs of consumers [5]. These data, which record consumers' data in an all-round and three-dimensional way, are called user portraits. Referring to the user interest model created by Pazzani and Billsus and the customer segmentation method oriented to marketing, this paper proposes a group user portrait construction method based on the order data of e-commerce platform, which is divided into four steps [4]. Firstly, the novelty of the selected variables, attributes and determined value range is that it can be defined according to the subdivision dimension of user portrait and combined with the shopping characteristics of online store customers, so as to better improve the accuracy of user portrait analysis of e-commerce platform. Therefore, a user portrait template is created for e-commerce platform stores through users' basic attributes and domain vocabulary. The main task of user avatar template is to select variables representing specific attributes of user avatar, and determine the type and value range of these attribute variables [6]. According to the segmentation dimension of user portrait, combined with the shopping characteristics of online store customers, we define the user portrait template as a four tuple, including user basic attribute set, product function attribute set, and product performance attribute set and product social attribute set [7]. The elements in the whole user portrait collection are defined based on the vocabulary of the user's basic attribute set, such as age, gender and address. The specific number depends on the detail of the customer information collected in the e-commerce platform. After determining the attributes of the selected user profile, the attribute variables should be defined, including the type and the corresponding value range. Attribute types are divided into quantitative attributes and qualitative attributes, mainly qualitative attributes, but both need quantitative characterization [8]. Quantitative attributes refer to numerical variables, such as price, purchase quantity, etc. Qualitative attributes mainly include sub type variables and ordinal variables, such as gender, region and clothing color, which belong to sub type variables. Product quality and grade are ordinal variables. Secondly, create portrait instances one by one according to the store order data, that is, single user portrait instances, and form a user portrait database [9]. Then, the attributes of single user portrait are normalized, that is, the attributes of different variable types are normalized to facilitate the subsequent attribute similarity calculation. For numerical attribute variables, the linear function transformation method is used for normalization. The process can be expressed as follows:

$$
B=\left|\frac{A-A_{\min }}{A_{\max }-A_{\min }}\right|
$$

In Formula (1), $A$ represents the attribute variable before conversion; $B$ represents the transformed attribute variable; $A_{\max }$ indicates the maximum value of the value range of the attribute; $A_{\min }$ represents the minimum value of the range of attribute values. Ordinal attribute variables, classified by grade, have a certain order of values. Therefore, ordinal attribute variables can be transformed into discrete values between $[0,1]$ according to the number of grades. The quantitative calculation formula can be expressed [10] as follows:

$$
B=\frac{2 x-1}{2 y}
$$

In Formula (2), $x$ represents the ordering of attribute variables; $y$ is the number of grades. The quantification of categorical attribute variables is complex, which can be transformed into multi-dimensional quantitative values by constructing conceptual classification dimension. Finally, for the group user portrait clustering, through attribute similarity calculation, determine the number of clusters, realize the e-commerce platform store user segmentation, and provide the basis for the subsequent purchase behavior preference analysis. 


\section{B. Feature Extraction of Consumer Purchase}

\section{Behavior Based on Data Mining Algorithm}

In order to better reflect consumers' shopping habits and provide basis for subsequent user product recommendation, it is also necessary to extract consumers' purchase behavior characteristics by using data mining algorithm on the basis of creating user portrait template of e-commerce platform. The features used for modeling can be divided into four categories: basic features, ranking features, label features and average weighted selection propensity features [10]. The basic features are divided into original features and combined features. Original features refer to the characteristics of the data set itself, such as click frequency, purchase frequency, etc. Combination feature is to produce new features with practical significance through combination and transformation of original features, such as purchase conversion rate, proportion of shopping cart, etc. The ranking feature is the ranking of the frequency of various behaviors of users on goods in the category of goods [11]. The label feature is whether the user has any corresponding operation on the goods within 3 days before the inspection date. In order to overcome the shortcoming that the implicit feedback data does not indicate the user's preference, the average weighted selection tendency is constructed by using the exhaustive method combined with a variety of user behavior data. This paper attempts to establish six feature groups, namely user level, commodity level, category level, user commodity level, user category level, commodity category level, and then uses data mining algorithm to filter features. When selecting features, the following two aspects can be considered. First, we can observe whether the features diverge. If the feature does not diverge, there is almost no difference in the sample, and the feature hardly works in the process of distinguishing samples [12]. Secondly, we can consider whether there is a high correlation between target and feature. If there is a high correlation between the two, priority can be given. Through the training of data mining algorithm, the weights of different features are obtained. The idea of feature importance evaluation in data mining algorithm is to judge the contribution of each feature, then take the average value, and finally compare the contribution between features [13]. Among them, the calculation method of contribution is Gini index. Gini index is calculated by the formula:

$$
G=1-\sum_{k=1}^{k} g^{2}
$$

In Formula (3), $G$ is Gini index; $k$ means category; $g$ is sample weight. The importance of features at a node can be expressed by the change of Gini index before and after node branching [14]. The calculation formula is as follows:

$$
V=G_{0}-G_{1}-G_{2}
$$

In Formula (4), $V$ represents the importance of features at a node; $G_{0}$ is the Gini index before the split;
$G_{1}, G_{2}$ represent the Gini index of the two new nodes after the split. By normalizing the importance scores of all nodes, we can get the importance of the feature, and then rank the feature importance. Feature importance ranking uses Python S cikit-learn library. We first load the RandomForestClassifier module in the $\mathrm{S}$ cikit-learn library to model all the data. After the model is built, we use the feature_importances_function in the module to output the importance of all features. This function will calculate the Gini coefficient change value of each node that is, after feature splitting, and record it. Finally, the importance of each feature can be obtained by making a unified normalization change [14]. Removing the lower ranking features through feature selection can speed up the operation of the next purchase behavior preference analysis model, improve the evaluation accuracy of the model, and improve the overall performance of the model.

\section{Design the Clustering Algorithm of Consumer}

\section{Purchase Behavior}

When studying user behavior, dividing user behavior and clustering user purchase behavior can better mine the behavior characteristics of user groups and master their preference rules, so as to guide the repair and iteration of subsequent e-commerce platform services. In the past, many scholars used traditional clustering algorithms such as k-means and EM to subdivide the user's purchase behavior. These traditional algorithms are simple and intuitive. However, although widely used, this kind of traditional clustering algorithm has some defects. For example, the clustering results may fall into the problem of local optimum, which makes the output unstable and is not conducive to the subsequent study of behavior preference. In view of the shortcomings of traditional clustering algorithm, the spectral clustering algorithm selected in this paper has the characteristics of clustering on arbitrary shape sample space and converging to the global optimal solution [15]. Spectral clustering constructs a new data space by introducing Gaussian kernel function to map the original data to infinite dimension, and divides the complex data structure clusters by hyperplane in the new data space. Among them, the multi-channel spectral algorithm is used for multi-channel segmentation, and the algorithm only needs one time to realize the division of multiple subgraphs, and the calculation process is more efficient. Therefore, this paper will use NJW algorithm (A multi-channel spectral clustering algorithm proposed by $\mathrm{Ng}$ et al.), which is the classic multi-channel spectral algorithm, and improve it on the basis of NJW algorithm to obtain better clustering results. The intrinsic gap is introduced to determine the number of clusters in NJW algorithm. Intrinsic gap refers to the difference between adjacent eigenvalues. According to the matrix perturbation theory, the larger the eigengap is, the more stable the subspace composed of the selected eigenvectors is [16]. Based on this, the subscript corresponding to the first maximum value in the intrinsic gap sequence is the value of clustering data needed by the algorithm. The introduction of intrinsic gap 
theory can effectively improve the clustering performance of the algorithm. Based on the above theoretical analysis, the flow of the improved NJW algorithm is shown in Figure 1.

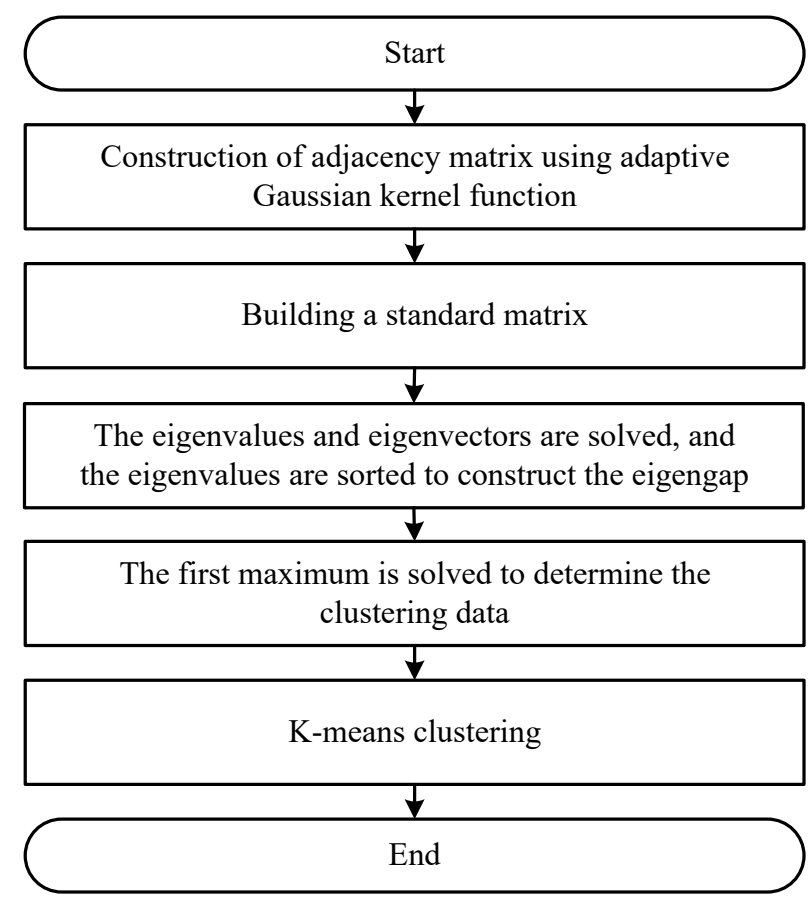

Figure 1. Flow chart of improved NJW algorithm

Taking the characteristics of consumers' purchasing behavior extracted above as the research object of cluster analysis, the purchasing behavior clustering is carried out according to the following steps. Step 1: construct undirected weight graph of samples and calculate similarity matrix. The calculation formula is as follows:

$$
R=\left\{\begin{array}{l}
\exp \left(\frac{-\left\|a_{m}-a_{n}\right\|^{2}}{\varepsilon_{m} \varepsilon_{n}}\right), m \neq n \\
0, m=n
\end{array}\right.
$$

In Formula (5), $R$ represents the similarity matrix; $\exp$ is an exponential function based on the natural constant e; $a_{m}, a_{n}$ represent sample data; $\varepsilon_{m}, \varepsilon_{n}$ are the Gaussian kernel function parameter of the sample; $m, n$ are the sample serial number. Step 2: calculate the standardized Laplacian matrix of the sample data set. Step 3: extract the corresponding eigenvalues and eigenvectors of the matrix according to the standardized Laplacian matrix, and sort the eigenvalues of the matrix in descending order according to the matrix perturbation theory. At the same time, the intrinsic gap sequence is constructed and the subscript of the first maximum value in the sequence is found as the $\mathrm{k}$ value of the cluster, so as to optimize the limitations of the artificial definition of the number of clusters [17], [18]. Step 4: select the first $\mathrm{k}$ largest eigenvalues and the corresponding eigenvectors according to the $\mathrm{k}$ value, and construct a new matrix, that is, map the original sample matrix to the new sample space, and then reduce the dimension, and carry out the unit processing for the new clustering sample space. Step 5: each row in the matrix is regarded as a point in the new sample space, and traditional clustering algorithms such as $\mathrm{k}$-means are used to cluster, and then $\mathrm{k}$ clusters are obtained. Step 6: output k clusters of sample data set. So far, the clustering of consumer purchase behavior is completed.

\section{Establish the Model of Consumer's Purchase}

\section{Behavior Preference}

Through the design of clustering algorithm to achieve the segmentation of consumer purchase behavior, on this basis, the consumer purchase behavior preference model is established to predict and evaluate the consumer purchase behavior of e-commerce platform. Before constructing the purchase behavior preference model, it is necessary to separate the characteristic variables in advance, that is, discretize the continuous variables [19]. Through the data frame of variables, the subsequent classification model can be simplified, so as to reduce the risk of over fitting. At the same time, data partitioning can also improve the overall stability of the model. Because the consumer data of e-commerce platform will constantly change, the setting of variable interval can effectively avoid the situation that the evaluation of the consumer changes greatly due to the data update. In addition, the discretization of the data also helps to improve the robustness of the model to abnormal data in consumer purchasing behavior. In this paper, the variable WOE coding method is used to process the data in discrete boxes. The calculation formula of WOE value [20] is as follows:

$$
w=\ln \left(\frac{s_{i}}{u_{i}} / \frac{s}{u}\right)
$$

In Formula (6), $w$ is the value of WOE; $i$ is the variable; $s_{i}$ is the number of sample 1 corresponding to the variable; $u_{i}$ is the number of samples corresponding to the variable; $s$ is the number of sample $1 ; u$ is the number of samples 0 . By combining the WOE code and the fitting coefficient of purchase behavior preference model, the final analysis results can be transformed into the form of score card. Because the model finally needs to transform the comprehensive indicators into e-commerce platform consumers' purchase behavior preference, it is necessary to set the conversion rules of model indicators to build the behavior preference evaluation model [20]. In this paper, with the help of the score card conversion method, the Lasso Logistic model input is combined with the WOE (Weight of Evidence) code to carry out the score conversion through rule conversion. According to the general scoring card model, the score conversion rules of logistic regression are as follows:

$$
c=\alpha-\beta \log \left(\frac{p}{1-p}\right)
$$

In Formula (7), $c$ is the fraction obtained after 
transformation; $\alpha$ and $\beta$ are manually set constants; $p$ is the probability that the consumer has a purchase preference. Therefore, the formula shows that the higher the probability of consumers' purchase preference, the higher the score. According to the variable score card of consumers' purchase behavior, each consumer in the data is scored on the characteristics of the consumption process of e-commerce platform [21]. After the corresponding score of each consumer is obtained, the score is divided by the highest score obtained from the behavior preference model, and then the score is finally converted into the form of percentage (rounded down), which is used as the index of consumer behavior preference for e-commerce platform consumption. Based on the above process, this paper designs the analysis method of consumer purchase behavior preference of e-commerce platform based on data mining algorithm.

\section{EXPERIMENT}

\section{A. Experimental Preparation}

In order to test the application effect of e-commerce platform consumer purchase behavior preference analysis method based on data mining algorithm, the following experimental verification. The data set in this paper comes from the Tianchi community of Ali AI. The data set includes about 130 million user behaviors of about 1.5 million random users who have consumption behaviors in December 2020 (including browsing, purchasing, adding shopping cart and collecting, etc.). Each row of the dataset represents a user behavior, which is composed of user ID, commodity ID, commodity category ID, behavior type and timestamp, separated by commas. Limited to the local computer hardware configuration, in order to save processing time, 25000 users were randomly selected from the 1500000 users of the original data, and 2165287 user behavior data were retained. Because the consumer behavior data of e-commerce platform users also includes the data generated by crawler users and swipe users, the behavior of these two types of users is obviously different from the normal user behavior, which will interfere with the observation of normal user behavior, so it is necessary to eliminate them. Through data observation, we can find that some users in December 2020 only browse behavior, no purchase behavior, such users are suspected of crawler users. Some users' browsing, collecting and adding shopping cart records are all 0 , but they have purchase records, which are suspected of swiping orders. After excluding the 1324 crawler users, 64 swipe users and all the behaviors of these two types of users, there are 23612 users and their 1832174 purchase behaviors. Taking the cleaned data set as the research sample, an experiment is designed to measure the performance of the purchase behavior preference analysis method [22].

\section{B. Experimental Result}

In order to evaluate the superiority of the analysis method of purchasing behavior preference designed in this paper, it is compared with the existing analysis methods of purchasing behavior preference (the method proposed by Jiang et al. [12] and the method proposed by Pereira et al. [13]), and the evaluation accuracy of various methods on consumers' purchasing behavior preference is calculated. In this experiment, accuracy rate, recall rate and F1 value are used to evaluate the prediction effect of each method on consumers' purchase behavior preference. The data set is randomly divided into 10 sub sets, and the data of each sub set is used to predict consumers' purchase behavior preference. The experimental results are shown in Tables 1-3.

Table 1. Comparison results of accuracy rate (\%)

\begin{tabular}{cccc}
\hline $\begin{array}{c}\text { Serial } \\
\text { number }\end{array}$ & $\begin{array}{c}\text { Method } \\
\text { of this paper }\end{array}$ & $\begin{array}{c}\text { Existing } \\
\text { method 1 }\end{array}$ & $\begin{array}{c}\text { Existing } \\
\text { method 2 }\end{array}$ \\
\hline 1 & 91.26 & 64.44 & 78.36 \\
2 & 90.64 & 65.68 & 76.09 \\
3 & 91.87 & 66.22 & 75.54 \\
4 & 92.78 & 64.83 & 78.47 \\
5 & 91.52 & 65.06 & 76.75 \\
6 & 90.23 & 64.38 & 75.23 \\
7 & 92.35 & 63.55 & 78.93 \\
8 & 91.89 & 64.76 & 75.24 \\
9 & 92.61 & 65.93 & 76.56 \\
10 & 92.22 & 64.65 & 75.31 \\
\hline
\end{tabular}

Table 2. Comparison results of recall rate $(\%)$

\begin{tabular}{cccc}
\hline $\begin{array}{r}\text { Serial } \\
\text { number }\end{array}$ & $\begin{array}{c}\text { Method } \\
\text { of this paper }\end{array}$ & $\begin{array}{c}\text { Existing } \\
\text { method 1 }\end{array}$ & $\begin{array}{c}\text { Existing } \\
\text { method 2 }\end{array}$ \\
\hline 1 & 88.47 & 65.63 & 72.76 \\
2 & 89.65 & 65.56 & 71.25 \\
3 & 87.76 & 64.88 & 71.32 \\
4 & 88.23 & 66.22 & 72.37 \\
5 & 89.88 & 64.35 & 70.92 \\
6 & 89.32 & 64.47 & 71.61 \\
7 & 88.94 & 65.74 & 72.54 \\
8 & 87.21 & 66.99 & 70.46 \\
9 & 88.19 & 64.22 & 72.78 \\
10 & 89.08 & 65.40 & 71.83 \\
\hline
\end{tabular}

Table 3. Comparison results of F1 value (\%)

\begin{tabular}{cccc}
\hline $\begin{array}{r}\text { Serial } \\
\text { number }\end{array}$ & $\begin{array}{c}\text { Method } \\
\text { of this paper }\end{array}$ & $\begin{array}{c}\text { Existing } \\
\text { method 1 }\end{array}$ & $\begin{array}{c}\text { Existing } \\
\text { method 2 }\end{array}$ \\
\hline 1 & 89.84 & 65.03 & 75.46 \\
2 & 90.14 & 65.62 & 73.59 \\
3 & 89.77 & 65.54 & 73.37 \\
4 & 90.45 & 65.52 & 75.30 \\
5 & 90.69 & 64.70 & 73.72 \\
6 & 89.77 & 64.42 & 73.38 \\
7 & 90.61 & 64.63 & 75.60 \\
8 & 89.49 & 65.86 & 72.77 \\
9 & 90.35 & 65.06 & 74.62 \\
10 & 90.62 & 65.02 & 73.53 \\
\hline
\end{tabular}

According to the experimental results in Table 1 , the accuracy of this method is $91.74 \%$, which is $26.79 \%$ and $15.09 \%$ higher than the existing methods. According to the experimental results in Table 2, the recall rate of this method is $88.67 \%$, which is $23.32 \%$ and $16.89 \%$ higher than the existing methods. According to the experimental 
results in Table 3, the F1 value of this method is $90.17 \%$, which is $25.03 \%$ and $16.04 \%$ higher than the existing methods. From the above experimental results, it can be seen that the proposed method is superior to the existing analysis methods in terms of accuracy, recall rate and F1 value. A more accurate prediction result of consumer purchase behavior preference can be obtained, which can provide more personalized choices for e-commerce platform to push goods to consumers.

\section{CONCLUSION}

In order to improve the accuracy of consumer purchase behavior preference analysis in e-commerce platform, this paper studied a method of consumer purchase behavior preference in e-commerce platform based on data mining algorithm. Create a user profile template of e-commerce platform with consumer data record, select attribute variable and set value range. The paper used data mining algorithm to extract the purchasing behavior characteristics of user head image template, and took the characteristics as cluster analysis object, designed the clustering algorithm of consumer purchasing behavior, and grasped the common points of group behavior. On this basis, the consumer purchase behavior preference model was established to predict and evaluate the consumer purchase behavior preference. The experimental results showed that the accuracy rate of this method is $91.74 \%$, the recall rate is $88.67 \%$, and the $\mathrm{F} 1$ value is $90.17 \%$, which are higher than the existing methods, and can provide consumers with more satisfactory product information push. However, because this research mainly focuses on implicit feedback data mining, there are still some shortcomings because it does not combine explicit feedback data with implicit feedback data. Therefore, we will continue to study the relevant models in this field and apply better models to implicit feedback data mining through practice.

\section{REFERENCES}

[1] Gasimli V, Jiang M, Yuan X, Mammadov E, Ulubel $Y$. "The informational role of average rating and variance of customer ratings in the differential patterns of consumer behavior", Human Systems Management, vol. 39, no. 1, pp. 1-10, 2020.

[2] Viciunaite V, Alfnes F. "Informing sustainable business models with a consumer preference perspective", Journal of Cleaner Production, vol. 242, pp. 118417, 2020.

[3] Xiong X, Yuan F, Huang M, Cao M, Xiong XH. "Comparative evaluation of web page and label presentation for imported seafood products sold on Chinese e-commerce platform and molecular identification using DNA barcoding”, Journal of Food Protection, vol. 83, no. 2, pp. 256-265, 2020.

[4] Jiang H, Fan J. "Research and analysis of cigarette consumers' purchase behavior based on C4.5 algorithm", Journal of Ningbo University of Technology, no. 4, pp. 48-53, 2019.

[5] Qian M, Xu Z. Research on dynamic identification and effect verification of consumer brand decision preference based on machine learning. Nankai
Management Review, vol. 126, no. 3, pp. 68-78, 2019.

[6] Christopher A, Adler H, Pantri H. "Analysis of the influence of entrepreneurship capability, agility, business transformation, opportunity on start-up behavior in e-commerce companies in Indonesia during the Covid 19 pandemic", WSEAS Transactions on Business and Economics, vol. 18, pp. 1103-1112, 2021.

[7] Li H, Peng T. "How does heterogeneous consumer behavior affect pricing strategies of retailers?", IEEE Access, vol. 8, pp. 165018-165033, 2020.

[8] Kuzmanovic M, Savic G. "Avoiding the privacy paradox using preference-based segmentation: A conjoint analysis approach", Electronics, vol. 9, no. 9, pp. 1382, 2020.

[9] Soorani F, Ahmadvand M. "Determinants of consumers' food management behavior: Applying and extending the theory of planned behavior", Waste Management, vol. 98, no. 6, pp. 151-159, 2019.

[10]Cokun A, Zbük R M Y. "What influences consumer food waste behavior in restaurants? An application of the extended theory of planned behavior", Waste Management, vol. 117, no. 5, pp. 170-178, 2020.

[11] Mohanty P K, Dey D K. "Consumer-Brand engagement with e-commerce market place brands", Journal of Electronic Commerce in Organizations, vol. 18, no. 3, pp. 21-37, 2020.

[12] Jiang Y, Zhang JY, Wang YH, Wang WY "Capturing ownership behavior of autonomous vehicles in Japan based on a stated preference survey and a mixed logit model with repeated choices", International Journal of Sustainable Transportation, vol. 13, no. 10, pp. 788-801, 2019.

[13] Pereira M A, Figueira J R, Rui C M. "Using a Choquet integral-based approach for incorporating decision-maker's preference judgments in a Data Envelopment Analysis model", European Journal of Operational Research, vol. 284, no. 3, pp. 1016-1030, 2020.

[14] Raman P. "Online shopping characteristics and their influence on female buying behavior: An extension of the theory of planned behavior", Journal of Electronic Commerce in Organizations, vol. 18, no. 4, pp. 1-29, 2020.

[15] Yadav R, Mahara T. "Exploring the role of E-Servicescape dimensions on customer online shopping: A stimulus-organism-response paradigm", Journal of Electronic Commerce in Organizations, vol. 18, no. 3, pp. 53-73, 2020.

[16] Yu J, Shi W, Fang Y. "Construction of low carbon supply chain profit model considering consumer preference", Procedia CIRP, 2019, vol. 83, pp. 690-693.

[17] Kose T, Arslan S. Turkish consumer participation in e-commerce. Journal of Electronic Commerce in Organizations, vol. 18, no. 4, pp. 30-50, 2020.

[18] Lv Q. "Supply chain decision-making of cross-border e-commerce platforms", Advances in Industrial Engineering and Management, vol. 7, no. 1, pp. 1-5, 2018.

[19] Mccloskey D W, Koch S. "An examination of the data quality of online reviews: Who do consumers 
trust?", Journal of Electronic Commerce in Organizations, vol. 19, no. 1, pp. 24-42, 2021.

[20] Gupta P, Prashar S, Parsad C, Vijay TS. "Role of shopping app attributes in creating urges for impulse buying: An empirical investigation using SEM and neural network technique", Journal of Electronic Commerce in Organizations, vol. 19, no. 1, pp. 43-64, 2021.

[21] Veronika Y. Chernova, E. Degtereva, "Forecast of Development of the Dual-Use Industrial Products Market", WSEAS Transactions on Business and Economics, vol. 17, pp. 987-992, 2020.

[22] Aygun G E, Problems of Financial and Investment Support Modelling of the Regional Social and Economic Development, WSEAS Transactions on Business and Economics, vol. 17, pp. 741-752, 2020.
Creative Commons Attribution License $\mathbf{4 . 0}$ (Attribution 4.0 International, CC BY 4.0)

This article is published under the terms of the Creative Commons Attribution License 4.0

https://creativecommons.org/licenses/by/4.0/deed.en_US 